\title{
Opportunistic Access Schemes for Multiuser OFDM Wireless Networks
}

\author{
Cédric Gueguen and Sébastien Baey \\ Université Pierre et Marie Curie (UPMC) - Paris 6
}

France

\section{Introduction}

Bandwidth allocation in next generation broadband wireless networks (4G systems) is a challenging issue. The scheduling shall provide mobile multimedia transmission services with an adequate QoS. These new multimedia services with tight QoS constraints require increased system capacity together with high fairness. The past decades have witnessed intense research efforts on wireless communications. In contrast with wired communications, wireless transmissions are subject to many channel impairments such as path loss, shadowing and multipath fading. These phenomena severely affect the transmission capabilities and in turn the QoS experienced by applications, in terms of data integrity but also in terms of the supplementary delays or packet losses which appear when the effective bit rate at the physical layer is low.

Among all candidate transmission techniques for broadband transmission, Orthogonal Frequency Division Multiplexing (OFDM) has emerged as the most promising physical layer technique for its capacity to efficiently reduce the harmful effects of multipath fading. This technique is already widely implemented in most recent wireless systems like $802.11 \mathrm{a} / \mathrm{g}$ or 802.16 . The basic principle of OFDM for fighting the effects of multipath propagation is to subdivide the available channel bandwidth in sub-frequency bands of width inferior to the coherence bandwidth of the channel (inverse of the delay spread). The transmission of a high speed signal on a broadband frequency selective channel is then substituted with the transmission on multiple subcarriers of slow speed signals which are very resistant to intersymbol interference and subject to flat fading. This subdivision of the overall bandwidth in multiple channels provides frequency diversity which added to time and multiuser diversity may result in a very spectrally efficient system subject to an adequate scheduling.

\section{Resource Allocation in a Multiuser OFDM Wireless Access Network}

In this chapter, we focus on the proper allocation of radio resources among the set of mobiles situated in the coverage zone of a wireless access point in a centralized approach (Fig. 1). The packets originating from the backhaul network are buffered in the access point 
which schedules the downlink transmissions. In the uplink, the mobiles signal their traffic backlog to the access point which builds the uplink resource mapping.

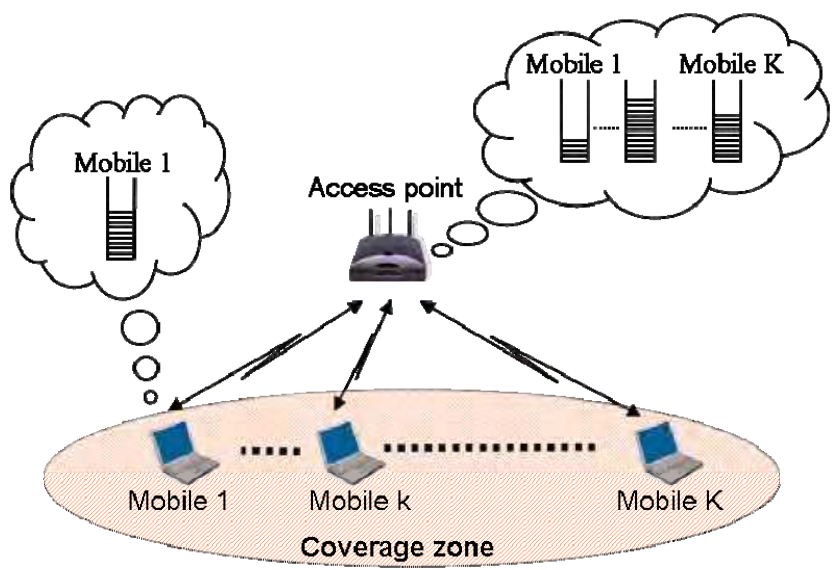

Fig. 1. Allocation of radio resources among the set of mobiles situated in the coverage zone of an access point.

The physical layer is operated using the frame structure described in Fig. 2. This structure is typical of OFDM wireless access networks like the OFDM mode of the IEEE 802.16-2004 (Hoymann, 2005). The total available bandwidth is divided in sub-frequency bands or subcarriers. The radio resource is further divided in the time domain in frames. Each frame is itself divided in time slots of constant duration. The time slot duration is an integer multiple of the OFDM symbol duration. The number of subcarriers is chosen so that the width of each sub-frequency band is inferior to the coherence bandwidth of the channel. Moreover, the frame duration is fixed to a value much smaller than the coherence time (inverse of the Doppler spread) of the channel. With these assumptions, the transmission on each subcarrier is subject to flat fading with a channel state that can be considered static during each frame.

The elementary resource unit (RU) is defined as any (subcarrier, time slot) pair. Each of these RUs may be allocated to any mobile with a specific modulation order. Transmissions performed on different RUs by different mobiles have independent channel state variations (Andrews et al., 2001). On each RU, the modulation scheme is QAM with a modulation order adapted to the channel state between the access point and the mobile to which it is allocated. This provides the flexible resource allocation framework required for opportunistic scheduling.

The system is operated using time division duplexing with four subframes: the downlink feedback subframe, the downlink data subframe, the uplink contention subframe and the uplink data subframe. The uplink and downlink data subframes are used for transmission of user data. In the downlink feedback subframe, the access point sends control information towards its mobiles. This control information is used for signalling to each mobile which RU(s) it has been allocated in the next uplink and downlink data subframes, the modulation order 
selected for each of these RUs and the recommended emission power in the uplink. In the uplink contention subframe, the active mobiles send their current traffic backlog and information elements such as QoS measures and transmit power. The uplink contention subframe is also used by the mobiles for establishing their connections. This frame structure supposes a perfect time and frequency synchronization between the mobiles and the access point as described in (Van de Beek et al., 1999). Therefore, each frame starts with a long preamble used for synchronisation purposes. Additional preambles may also be used in the frame.

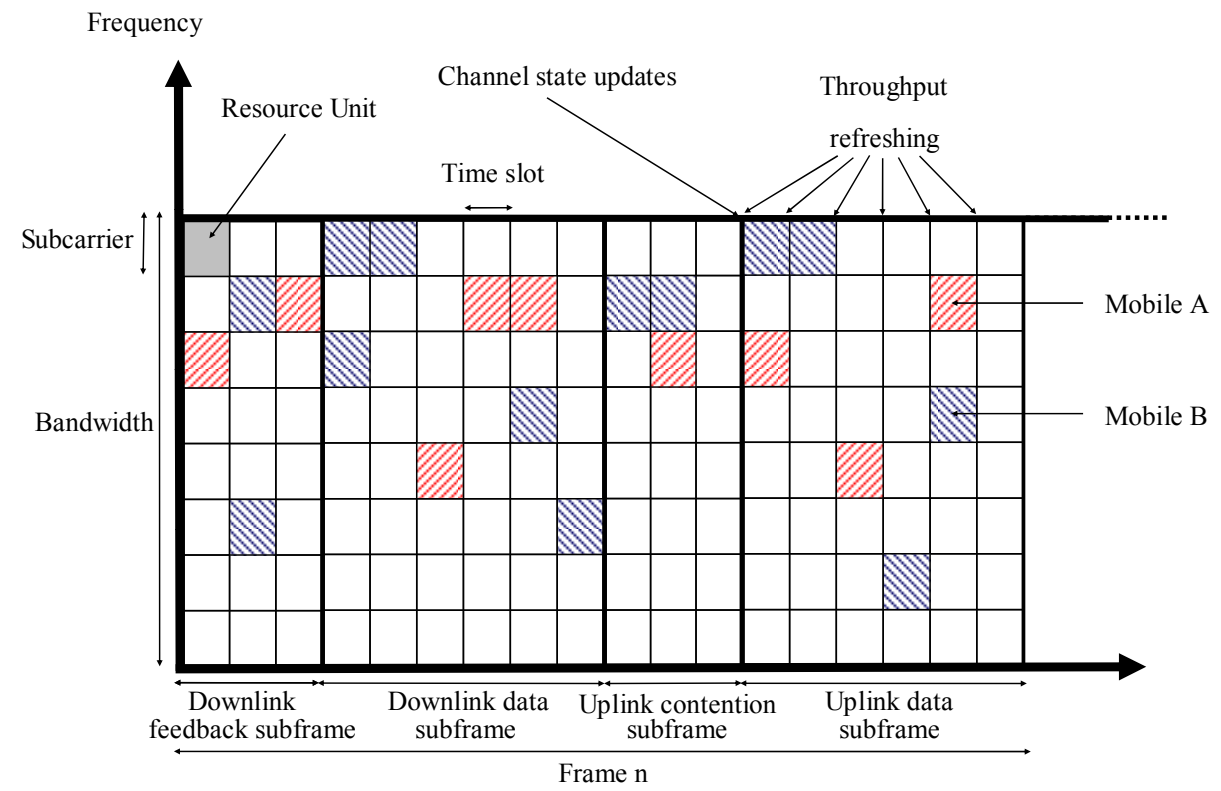

Fig. 2. Frame structure in TDD mode.

\section{Scheduling Techniques in OFDM Wireless Networks}

The MAC protocols currently used in wireless local area networks were originally and primarily designed in the wired local area network context. These conventional access methods like Round Robin (RR) and Random Access (RA) are not well adapted to the wireless environment and provide poor throughput. More recently intensive research efforts have been given in order to propose efficient schedulers for OFDM based networks and especially opportunistic schedulers which preferably allocate the resources to the active mobile(s) with the most favourable channel conditions at a given time. These schedulers take benefit of multiuser and frequency diversity in order to maximize the system throughput. All these schemes strongly rely on diversity for offering their good performances. Three major scheduling techniques have emerged: Maximum Signal-to-Noise Ratio (MaxSNR), Proportional Fair (PF) and recently, the Weighted Fair Opportunistic (WFO). 
Note that for these schedulers, knowledge of the channel state is supposed to be available at the receiver ( $\mathrm{Li}$ et al., 1999). The current channel attenuation on each subcarrier and for each mobile is estimated by the access node based on the SNR of the signal sent by each mobile during the uplink contention subframe. Assuming that the channel state is stable on a scale of $50 \mathrm{~ms}$ (Truman \& Brodersen, 1997), and using a frame duration of $2 \mathrm{~ms}$, the mobiles shall transmit their control information alternatively on each subcarrier so that the access node may refresh the channel state information once every 25 frames.

\subsection{Maximum Signal-to-Noise Ratio}

Many schemes are derived from the Maximum Signal-to-Noise Ratio (MaxSNR) technique (also known as Maximum Carrier to Interference ratio (MaxC/I)). In MaxSNR, priority is given at every scheduling event to the mobiles which have the greatest signal-to-noise ratio (SNR). It allocates the resource at a given time to the active mobile with the greatest SNR (Knopp \& Humblet, 1995; Wong et al., 1999; Wang \& Xiang, 2006). Denoting $m_{k, n}$ the maximum number of bits that can be transmitted on a time slot of Resource Unit $n$ if this RU is allocated to the mobile $k$, MaxSNR scheduling consists in allocating the RU $n$ to the mobile $j$ which has the greatest $m_{k, n}$ such as:

$$
j=\arg \max _{k}\left(m_{k, n}\right), k=1, \ldots, K
$$

Taking profit of multiuser and frequency diversity, MaxSNR scheduling continuously allocates the radio resource to the mobile with the best spectral efficiency. Consequently MaxSNR strongly increases the system throughput. Dynamically adapting the modulation and coding allows one to always make the most efficient use of the radio resource and come closer to the Shannon limit. However MaxSNR assumes that the user with the most favourable transmission conditions has information to transmit at the considered time instant. It does not take into account the variability of the traffic and the queuing aspects. Additionally, a negative side effect of this strategy is that the closest mobiles to the access point have disproportionate priorities over mobiles more distant since their path loss attenuation is much smaller. This results in a severe lack of fairness.

\subsection{Proportional Fair}

Proportional Fair (PF) algorithms have recently been proposed to incorporate a certain level of fairness while keeping the benefits of multiuser diversity (Viswanath et al., 2002; Kim et al., 2002; Anchun et al., 2003; Svedman et al., 2004; Kim et al., 2004). In PF based schemes, the basic principle is to allocate the bandwidth resources to a mobile $j$ when its channel conditions are the most favourable with respect to its time average such as:

$$
j=\arg \max _{k}\left(\frac{m_{k, n}}{M_{k, n}}\right), k=1, \ldots, K,
$$

where $M_{k, n}$ is the time average of the $m_{k, n}$ values. 
At a short time scale, path loss variations are negligible and channel state variations are mainly due to multipath fading, statistically similar for all mobiles. Thus, PF provides an equal sharing of the total available bandwidth among the mobiles as RR. Applying the opportunistic scheduling technique, system throughput maximization is also obtained as with MaxSNR. PF actually combines the advantages of the classical schemes and currently appears as the best bandwidth management scheme.

In PF-based schemes, fairness consists in guaranteeing an equal share of the total available bandwidth to each mobile, whatever its position or channel conditions. However, since the farther mobiles have a lower spectral efficiency than the closer ones due to pathloss, all mobiles do not all benefit of an equal average throughput despite they all obtain an equal share of bandwidth. This induces heterogeneous delays and unequal QoS. (Choi \& Bahk, 2007; Gueguen \& Baey (a), 2008; Holtzman, 2001) demonstrate that fairness issues persist in PF-based protocols when mobiles have unequal spatial positioning, different traffic types or different QoS targets. PF scheduling do not take into account the delay constraints and is not well adapted to multimedia services which introduce heterogeneous users, new traffic patterns with highly variable bit rates and stringent QoS requirements in terms of delay and packet loss.

\subsection{Weighted Fair Opportunistic}

More recently a new MAC scheduler, called "Weighted Fair Opportunistic (WFO)", has been proposed for efficient support of multimedia services in multiuser OFDM wireless networks (Gueguen \& Baey (b), 2008). Built in a higher layers/MAC/PHY cross-layer approach, this scheme is designed for best profiting of the multiuser diversity taking advantage of the dynamics of the multiplexed traffics. It takes into account both the transmission conditions and the higher layer constraints (traffic patterns, QoS constraints). In order to provide an efficient support of multimedia services, WFO dynamically favours the mobiles that go through a critical period with respect to their QoS requirements using dynamic priorities.

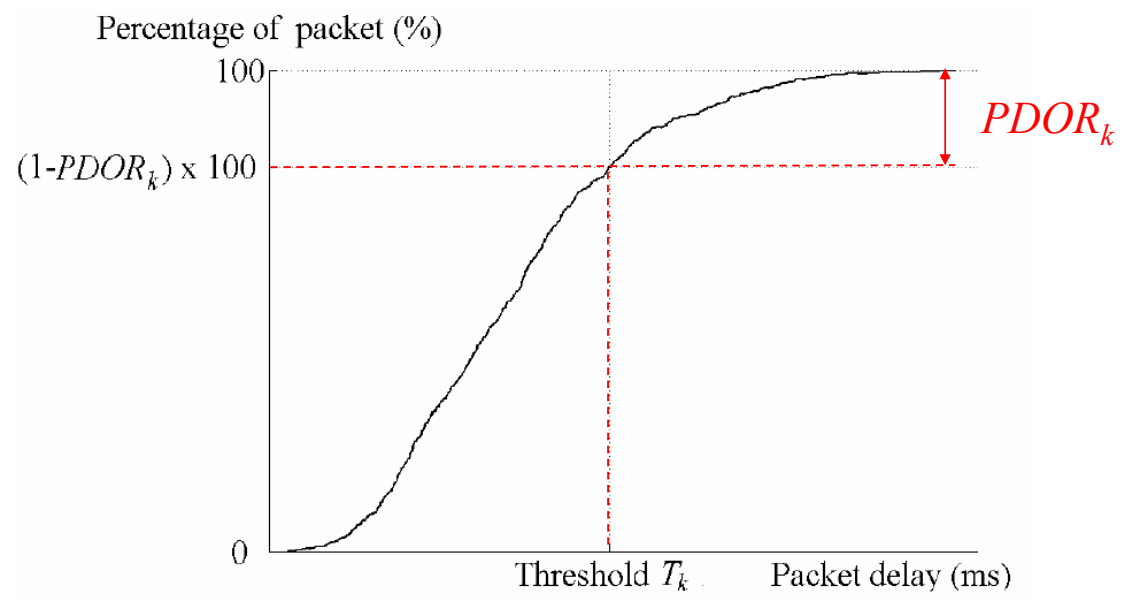

Fig. 3. Example packet delay CDF and experienced PDOR. 
Evaluating if a mobile goes through a critical period should not only focus on the classical mean delay and jitter analysis. Indeed, a meaningful constraint regarding delay is the limitation of the occurrences of large values. Accordingly, (Gueguen \& Baey (c), 2008) defines the concept of delay outage by analogy with the concept of outage used in system coverage planning. A mobile $k$ is in delay outage (in critical period) when its packets experience a delay greater than a given threshold $T_{k}$ defined by the mobile application requirements. The delay experienced by each mobile is tracked all along the lifetime of its connection. At each transmission of a packet of mobile $k$, the ratio of the total number of packets whose delay exceeded the threshold divided by the total number of packets transmitted since the beginning of the connection is computed. The result is called Packet Delay Outage Ratio (PDOR) of mobile $k$ and is denoted $P D O R_{k}$. This measure is representative of the emergency for the mobile $k$ to be served. Fig. 3 illustrates an example cumulative distribution of the packet delay of a mobile at a given time instant. A mobile can be considered as satisfied when, at the end of its connection, its delay constraint is met, i.e. its experienced PDOR is less than the application specific PDOR target.

In WFO scheduling, the required QoS, the experienced QoS and the transmission conditions are jointly considered in an extended cross-layer approach. The scheduling principle is to allocate a Resource Unit $n$ to the mobile $j$ which has the greatest WFO parameter value $W F O_{k, n}$ such as:

$$
j=\arg \max _{k}\left(W F O_{k, n}\right), k=1, \ldots, K
$$

where $W F O_{k, n}$ is defined by:

$$
W F O_{k, n}=m_{k, n} \times f\left(P D O R_{k}\right),
$$

with $f$ a strictly increasing polynomial function (Gueguen \& Baey (d), 2008; Gueguen \& Baey, 2009):

$$
f(x)=1+\beta \times x^{\alpha}
$$

The exponent parameter $a$ allows being sensitive and reactive to PDOR fluctuations which guarantees fairness at a short time scale. $\beta$ is a normalization parameter that ensures that $f\left(P D O R_{k}\right)$ and $m_{k, n}$ are in the same order of magnitude.

With this scheduling, physical layer information (represented through the factor $m_{k, n}$ ) are used in order to take advantage of the time, frequency and multiuser diversity and maximize the system capacity. Higher layer information (represented through the factor $f\left(P D O R_{k}\right)$ are exploited in order to introduce dynamic priorities between flows for ensuring the same QoS level to all mobiles. With this original weighted system that introduces dynamic priorities between the flows, WFO keeps a maximum number of flows active across time but with relatively low traffic backlogs. This results in a well-balanced resource allocation. Preserving the multiuser diversity allows to continuously take a maximal benefit 
of opportunistic scheduling and thus maximize the bandwidth usage efficiency. When the frequency diversity is sufficient, WFO better conceals the system capacity maximization, QoS support and fairness objectives than PF and MaxSNR schemes.

\section{Performance Evaluation}

In this section we compare the most acknowledged schedulers, the Round Robin (RR), the MaxSNR and the PF schemes with the most promising, the Weighted Fair Opportunistic (WFO) scheduling. Each is implemented with subcarrier by subcarrier allocation. Performance evaluation results are obtained using OPNET discrete event simulations. We focus on the main scheduling problem: maximize the system capacity while ensuring high fairness between mobiles localised at heterogeneous spatial positions in the cell.

In the simulations we assume 128 subcarriers and 5 time slots in a frame. The channel gain model on each subcarrier considers free space path loss and multipath Rayleigh fading (Parsons, 1992). We introduce a reference distance $d_{\text {ref }}$ for which the free space attenuation equals $a_{r e f}$. As a result the channel gain is given by:

$$
a_{k, n}=a_{r e f} \times\left(\frac{d_{r e f}}{d_{k}}\right)^{3.5} \times \alpha_{k, n}^{2},
$$

where $d_{k}$ is the distance to the access point of the mobile $k$ and $a_{k, n}^{2}$ represents the flat fading experienced by this mobile $k$ if it transmits or receives on subcarrier $n$. In the following, $a_{k, n}^{2}$ is Rayleigh distributed with an expectancy equal to unity.

The maximum transmit power satisfies:

$$
10 \log _{10}\left(\frac{P_{\max } \times T_{s}}{N_{0}} \times a_{r e f}\right)=31 \mathrm{~dB},
$$

where $T_{s}$ is the time duration of an OFDM symbol, $P_{\max }$ is the maximum achievable transmit power and $N_{0}$ is the single-sided power spectral density of noise. The BER target is taken equal to $10^{-3}$. With this setting, the value of $m_{k, n}$ for the mobiles situated at the reference distance is 6 bits when $a_{k, n}^{2}$ equals unity.

We assume all mobiles run the same videoconference application. This demanding type of application generates a high volume of data with high sporadicity and requires tight delay constraints, which substantially complicate the task of the scheduler. Each mobile has only one service flow with traffic composed of an MPEG-4 video stream (Baey, 2004) and an AMR voice stream (Brady, 1969).

In these extended simulations, we analyzed the behaviour of the schedulers when mobiles occupy different geographical positions. The objective is to clearly exhibit the ability of the 
opportunistic schedulers to provide fairness whatever the respective position of the mobiles. We first study a general context that includes mobility. We constitute two groups of 7 mobiles that both move straight across the cell, following the pattern described in Fig. 4 and Fig. 5 . Each mobile has a speed of $3 \mathrm{~km} / \mathrm{h}$ and the cell radius is taken equal to $5 \mathrm{~km}\left(3 d_{\text {ref }}\right)$. When a group of mobiles comes closer to the access point, the other group simultaneously goes farther away. Additionally, the threshold time $T_{k}$ is fixed to the value $80 \mathrm{~ms}$ in order to consider real time constraints and the PDOR target is $5 \%$.

Fig. 4. Mobility pattern.

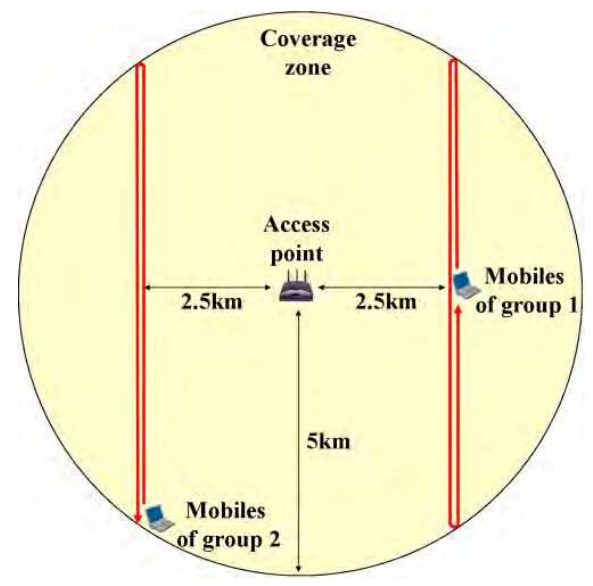

Considering the path loss, the Rayleigh fading and this mobility model, we have computed in Fig. 5 the evolution of the mean number of bits that may be transmitted per Resource Unit for each group of mobiles, averaging over all the Resource Units of a frame. This shows the impact of the mobile position on the mean $m_{k, n}$ values.

Regarding fairness, in wireless networks, it is well known that the closest mobiles to the access point generally obtain better QoS than mobiles more distant thanks to their higher spectral efficiency. Fig. 6 reports the mean PDOR experienced by each group of mobiles across the time. MaxSNR is highly unfair. Indeed, as soon as the mobiles move away from the access point, they experience high delays with a high number of packets in delay outage. $\mathrm{PF}$ offers better results. It brings more fairness and globally attenuates the delay peaks of the critical periods. However, we observe that WFO is the one that best smoothes these peaks. It adequately and continuously allocates the adequate priorities between the mobiles reacting to their relative movement across the cell. Providing a totally fair allocation of the bandwidth resources, the WFO scheduling smoothes the delay experienced by each mobile across time. Consequently, it further enhances the PF performances and the PDOR values are further decreased. WFO results in a very fair resource allocation that fully satisfies the delay constraints whatever the movement of the mobile. 

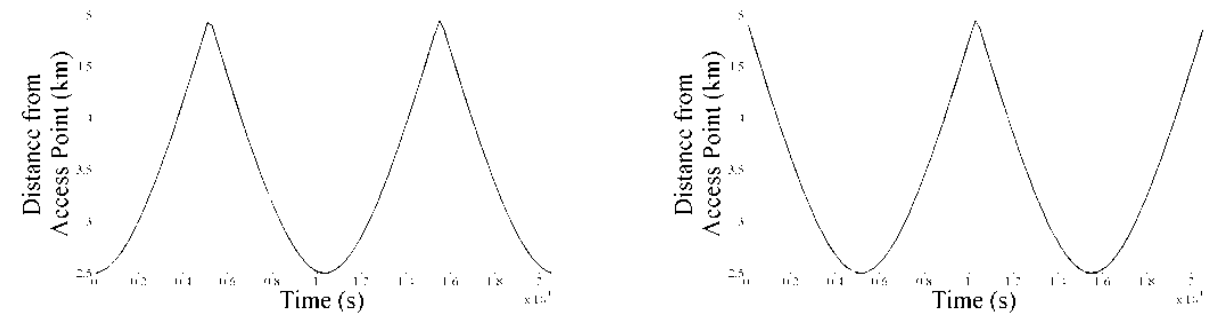

Fig. 5. Position of the mobiles across time (for mobiles of group 1 on the left and for mobiles of group 2 on the right).
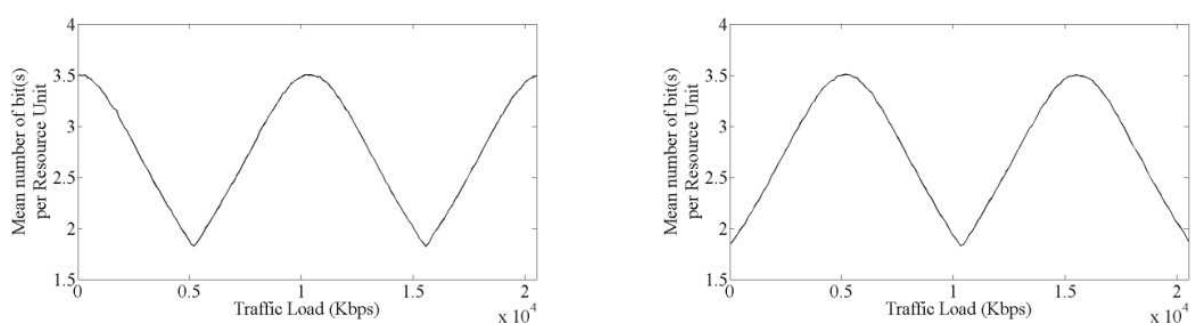

Fig. 6. Mean number of bit(s) per Resource Unit for each group of mobiles (for mobiles of group 1 on the left and for mobiles of group 2 on the right).
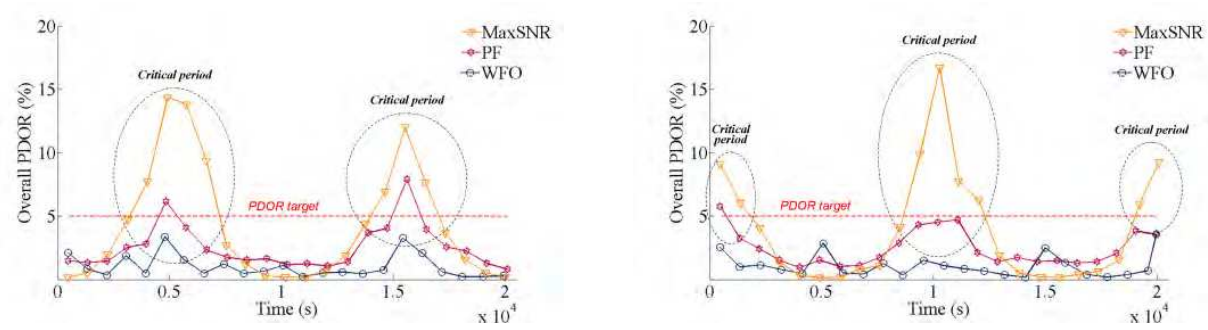

Fig. 7. PDOR fluctuation experienced by each group of mobiles (for mobiles of group 1 on the left and for mobiles of group 2 on the right).

In order to further underline the advantage of opportunistic schedulers compared to the classical Round Robin, we now study precisely the performance of the algorithms in a subscenario where all mobiles are static. A first half of mobiles are situated close to the access point and a second half 1.5 farther. The other parameters are identical for all the mobiles as described in Table 1 . The total number of mobiles sets the traffic load.

\begin{tabular}{|c|c|c|c|}
\hline Group & Distance $d_{k}$ & Delay threshold $T_{k}$ & Data rate \\
\hline 1 & $2 d_{\text {ref }}$ & $80 \mathrm{~ms}$ & $80 \mathrm{Kbps}$ \\
\hline 2 & $3 d_{\text {ref }}$ & $80 \mathrm{~ms}$ & $80 \mathrm{Kbps}$ \\
\hline
\end{tabular}

Table 1. Scenario setup with static mobiles. 
First we focus on the fairness provided by each scheduler. Fig. 7a, 7b, 7c and 7d display the overall PDOR for different traffic loads considering the influence of the distance on the scheduling.

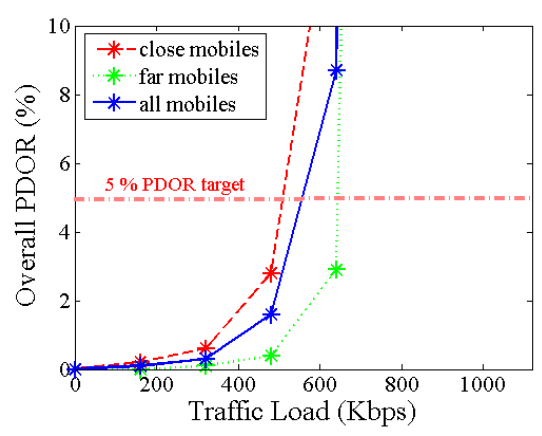

Fig. 8 a. With RR.

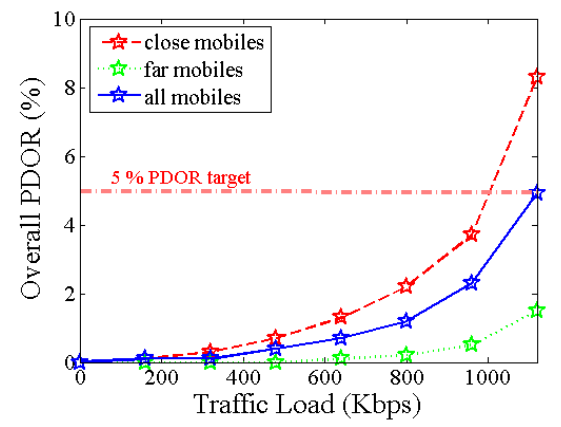

Fig. 8 c. With PF.

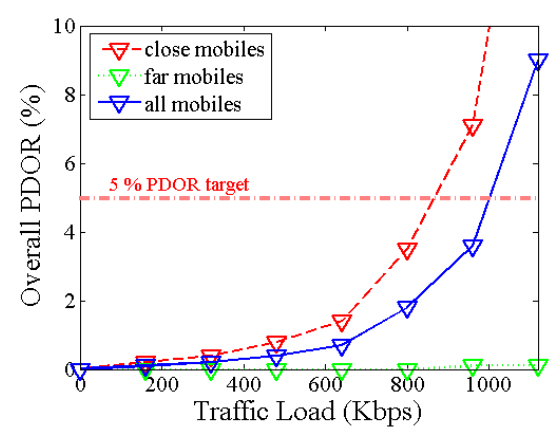

Fig. 8 b. With MaxSNR.

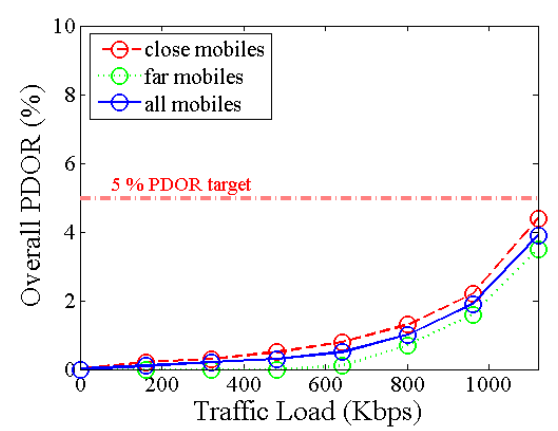

Fig. 8 d. With WFO.

Fig. 8. Measured QoS with respect to distance.

The classical RR fails to ensure the same PDOR to all mobiles. Actually, the RR fairly allocates the RUs to the mobiles without taking in consideration that far mobiles have a much lower spectral efficiency than closer ones. Moreover, the RR does not take benefit of multiuser diversity which results in a bad utilization of the bandwidth and in turn, poor system throughput. Consequently, an acceptable PDOR target of $5 \%$ is exceeded even with relatively low traffic loads. Based on opportunistic scheduling, the three other schemes globally show better QoS performances supporting a higher traffic load. However, MaxSNR and PF still show severe fairness deficiencies. Close mobiles easily respect their delay requirement while far mobiles experience much higher delays and go past the $5 \%$ PDOR target when the traffic load increases. In contrast, WFO provides the same QoS level to all mobiles whatever their respective position. WFO is the only one to guarantee a totally fair allocation. This allows reaching higher traffic loads with an acceptable PDOR for all mobiles. Additionally, looking at the overall PDOR for all mobiles at different traffic loads shows that, besides fairness, WFO provides a better overall QoS level as well. 


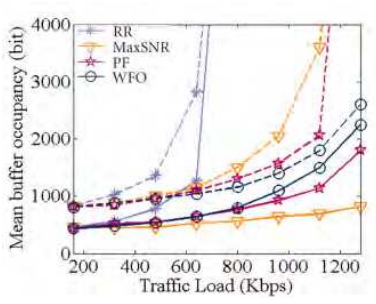

Fig. 9 a. Mean buffer occupancy for close mobiles (solid lines) and far mobiles (dashed lines).

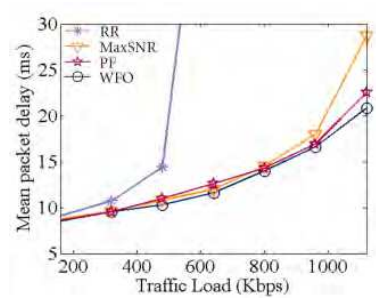

Fig. 9 b. Mean packet delay.

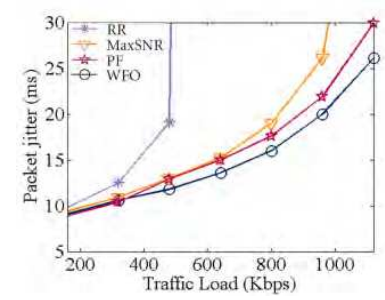

Fig. 9 c. Packet jitter.

Fig. 9. Buffer occupancy, delay and jitter.

Observing the mean buffer occupancy in Fig. 8a, WFO clearly limits the buffer occupancy to a same and reasonable value whatever the position of the mobile. This allows staying under the PDOR target for any traffic load. With its system of weights, WFO dynamically adjusts the relative priority of the flows according to their experienced delay. With this approach, sparingly delaying the closer mobiles, WFO builds on the breathing space offered by the easy respect of the delay constraints of the closer mobiles (with better spectral efficiency) for helping the farther ones. The WFO interesting performance results are corroborated in Fig. $8 \mathrm{~b}$ and $8 \mathrm{c}$ where the overall values of the mean packet delay and jitter obtained using WFO are smaller.

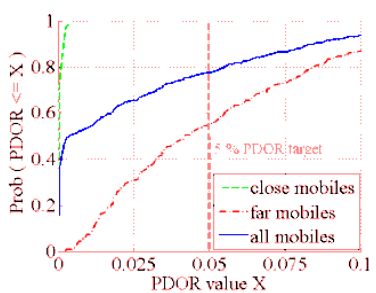

Fig. 10 a. CDF of end cycle PDOR with MaxSNR.

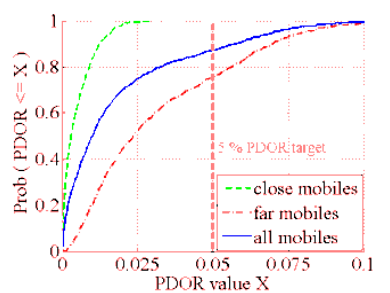

Fig. 10 b. CDF of end cycle PDOR with PF

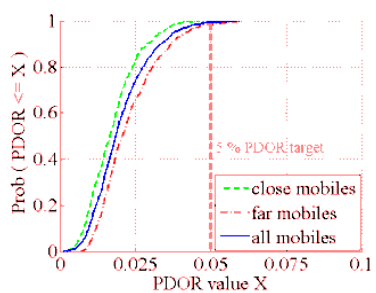

Fig. 10 c. CDF of end cycle PDOR with WFO

Fig. 10. Perceived QoS with different allocation schemes.

We then had a look at the QoS satisfaction level that each mobile perceives across the lifetime of a connection. We divided the connection of each mobile in cycles of five minutes and measured the PDOR at the end of each cycle. Fig. 9 shows the CDF of end cycle PDOR values for a traffic load of $960 \mathrm{Kbps}$, using respectively the MaxSNR, the PF and the WFO schemes (RR performances are not presented here since it is not able to support this high traffic load.). We also estimated the mobile dissatisfaction ratio. We checked if at the end of each cycle the delay constraint is met or not. We then computed the mobile dissatisfaction ratio defined as the number of times that the mobiles are not satisfied (experienced PDOR $\geq$ PDOR $_{\text {target}}$ ) divided by the total number of cycles (cf. Fig. 10). 

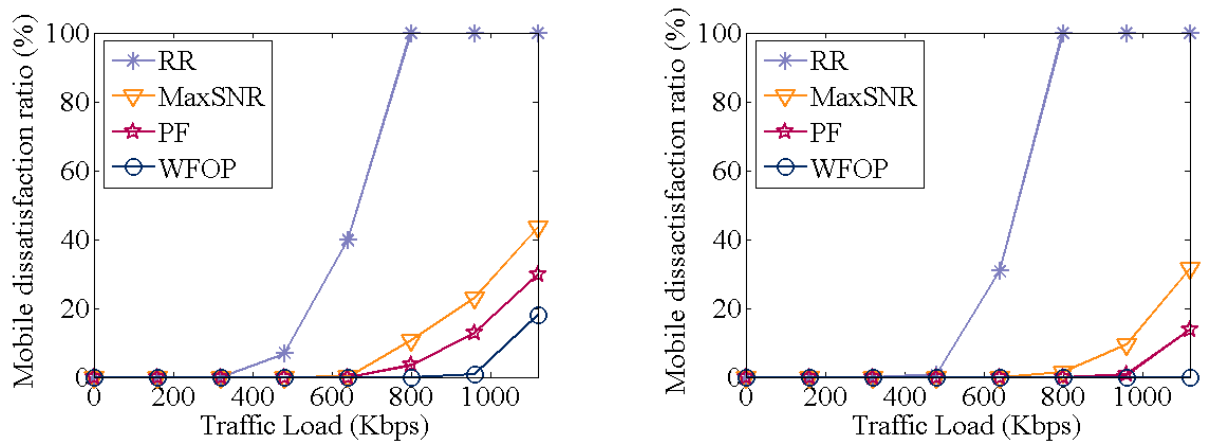

Fig. 11. Analysis of the respect of QoS constraints for different targeted QoS (Mobile dissatisfaction if $\mathrm{PDOR}_{\text {target }}=5 \%$ on the left, if $\mathrm{PDOR}_{\text {target }}=10 \%$ on the right).

Highly unfair, MaxSNR fully satisfies the required QoS of close mobiles at the expense of the satisfaction of far mobiles. Indeed, only 54.5 percents of these latter experience a final PDOR inferior to a PDOR target of $5 \%$ (cf. Fig. 9a). Unnecessary priorities are given to close mobiles who easily respect their QoS constraints while more attention should be given to the farther. This inadequate priority management dramatically increases the global mobile dissatisfaction, which reaches $23 \%$ as shown on Fig. 9a and Fig. 10 (on the left).

PF brings more fairness and allocates more priority to far mobiles. Compared to MaxSNR, PF offers a QoS support improvement with only $12.8 \%$ of dissatisfied mobiles (cf. Fig. 9b and Fig. 10 (on the right)). Fairness is still not total since the farther mobiles have a lower spectral efficiency than the closer ones due to pathloss. All mobiles do not all benefit of an equal average throughput despite they all obtain an equal share of bandwidth. This induces heterogeneous delays and unequal QoS. This fairness improvement compared to MaxSNR indicates however that some flows can be slightly delayed to the benefit of others without significantly affecting their QoS.

WFO was built on this idea. The easy satisfaction of close mobiles (with better spectral efficiency) offers a degree of freedom which ideally should be exploited in order to help the farther ones. WFO allocates to each mobile the accurate share of bandwidth required for the satisfaction of its QoS constraints, whatever its position. With WFO, only 0.8 percents of the mobiles are dissatisfied (cf. Fig. 9c and Fig. 10 (on the left)). Additionally, compared to Fig. 9a and Fig. 9b, Fig. 9c exhibits superimposed curves, which prove the WFO high fairness, included at short term.

Fig. 10 shows that WFO brings the largest level of satisfaction. Indeed, for a tight PDOR target of $5 \%$ (see on the left), the dissatisfaction ratio with a high traffic load of $1120 \mathrm{Kbps}$ is equal to $18 \%$ with WFO versus $29.7 \%$ with PF, the best of the other scheduling schemes. If we set the PDOR target to $10 \%$, the dissatisfaction ratio with a high traffic load of $1120 \mathrm{Kbps}$ is $0 \%$ with WFO versus $13.8 \%$ with the best of the other scheduling schemes (PF). 


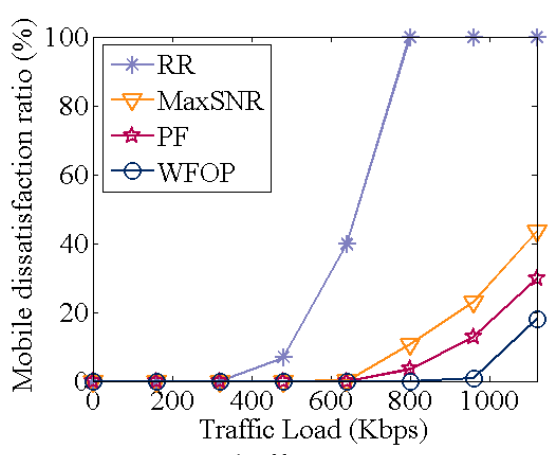

Fig. 12 a. Spectral efficiency.

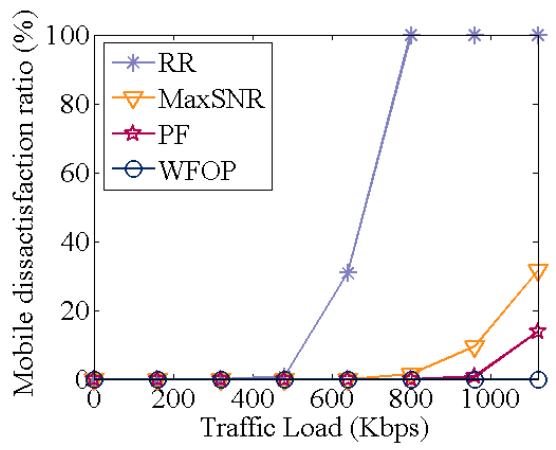

Fig. 12 b. Multiuser diversity

Fig. 12. Bandwidth usage efficiency.

We finally studied the system capacity offered by the four scheduling algorithms. Fig. 11a shows the average number of bits carried on a used subcarrier by each tested scheduler under various traffic loads. As expected, the non opportunistic Round Robin scheduling provides a constant spectral efficiency, i.e. an equal bit rate per subcarrier whatever the traffic load since it does not take advantage of the multiuser diversity. The three other tested schedulers show better results. In contrast with RR, with the opportunistic schedulers (MaxSNR, PF, WFO), we observe an interesting inflection of the spectral efficiency curve when the traffic load increases. The join analysis of Fig. 11a and Fig. 11b shows that the spectral efficiency of opportunistic scheduling is an increasing function of the number of active mobiles, thanks to the exploitation of this supplementary multiuser diversity. Consequently MaxSNR, PF and WFO increase their spectral efficiency with the traffic load and the system capacity is highly extended compared to networks which use classical scheduling algorithms. With these three schedulers, all mobiles are served even at the highest traffic load of $1280 \mathrm{Kbps}$.

The performance of the four schedulers can be further qualified by computing the theoretical maximal system throughput. Considering the Rayleigh distribution, it can be noticed that $a_{k, n}^{2}$ is greater or equal to 8 with a probability of only 0.002 . In these ideal situations, close mobiles can transmit/receive 6 bits per RU while far mobiles may transmit/receive 4 bits per RU. If the scheduler always allocated the RUs to the mobiles in these ideal situations, an overall efficiency of 5 bits per RU would be obtained which yields a theoretical maximal system throughput of $1600 \mathrm{Kbps}$. Comparing this value to the highest traffic load in Fig. 11a (1280 Kbps) further demonstrates the good efficiency obtained with the opportunistic schedulers that nearly always serve the mobiles when their channel conditions are very good. This result also shows that the WFO scheduling has slightly better performances than the two other opportunistic schedulers. Keeping more mobiles active (cf. Fig.11b) but with a relatively lower traffic backlog (cf. Fig.8a), the WFO scheme preserves multiuser diversity and takes more advantage of it obtaining a slightly higher bit rate per subcarrier (cf. Fig. 11a). 


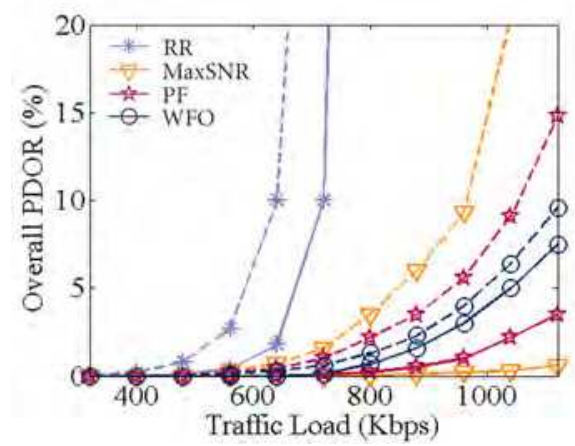

Fig. 13a. Measured QoS for close mobiles (solid lines) and far mobiles (dashed lines).

Fig. 13. Performances of schedulers with fixed multiuser diversity.

In the results described so far, the traffic load was varied by increasing or decreasing the number of mobiles in the system, which modified the multiuser diversity. This exhibited the opportunistic behaviour of the schedulers and especially their ability to take advantage of the multiuser diversity brought with the increase of the number of mobiles. We also studied the ability of each scheduler to take profit of the multiuser diversity brought by a given number of users. In Fig.12, we provide complementary results obtained in a context where the traffic load variation is done through just increasing the mobile bit rate requirement and keeping a constant number of users (10 mobiles). The results in Fig. 12a show that, as previously, WFO outperforms the other scheduling schemes. With its weighted algorithm, WFO dynamically adjusts the priorities of the mobiles and ensures a completely fair allocation. WFO is the only one which allows reaching higher traffic loads with an acceptable PDOR for all mobiles. Additionally, even if the traffic load increases without variation in the number of mobiles, WFO keeps more mobiles active across the time than the other schemes and takes better advantage of the multiuser diversity. The analysis of Fig. 12b confirms that WFO maximizes the average bit rate per subcarrier.

\section{Conclusion}

Opportunistic schedulers take benefit of multiuser and frequency diversity. They preferably allocate the resources to the active mobile(s) with the most favourable channel conditions at a given time. This maximizes the system throughput of OFDM wireless networks. Three major algorithms have emerged: MaxSNR, PF and more recently WFO. However, in spite of their high performances in terms of system throughput maximization, both MaxSNR and PF suffer of severe fairness deficiencies owing to unequal spatial positioning of the mobiles. This issue is resolved with WFO which appears as the best current opportunistic scheduler. WFO jointly considers the transmission conditions, the currently measured/experienced QoS and the QoS targets of the mobiles in the bandwidth allocation process. With an original weighted system that introduces dynamic priorities between the flows, it dynamically favors the flows that go through a critical period and always attributes the adequate priorities for improved QoS support. Keeping a maximum number of flows active 
across time but with relatively low traffic backlogs, WFO is designed for best profiting of the multi-user diversity taking advantage of the dynamics of the multiplexed traffics. Preserving the multiuser diversity, WFO takes a maximal benefit of the opportunistic scheduling technique and maximizes the system capacity. Additionally, this also achieves a time uniform fair allocation of the resource units to the flows ensuring short term fairness. This higher layers/MAC/PHY cross-layer approach better conceals the system capacity maximization, fairness objectives and the full support of multimedia services with adequate QoS.

\section{References}

Hoymann, C. (2005). Analysis and performance evaluation of the OFDM-based metropolitan area network IEEE 802.16. Computer Networks, Vol. 49, No. 3, pp. 341363, ISSN: 1389-1286

Andrews, M.; Kumaran, K.; Ramanan, K. Stolvar, A. \& whiting P. (2001). Providing quality of service over a shared wireless link. IEEE Communications Magazine, Vol. 39, No.2, pp. 150-154, ISSN: 0163-6804

Van de Beek, J.-J. ; Borjesson, P.O. ; Boucheret, M.-J ; Landstrom, D. ; Arenas, J.-M. Odling, P.; Ostberg, C.; Wahlgvist, M. \& Wilson, S.K. (1999). A time and frequency synchronization scheme for multiuser OFDM. IEEE J.Sel. Areas Commun, Vol.17, No.11, pp 1900-1914, ISSN: 0733-8716

Li, Y.G; Seshadri, N. \& Ariyavisitakul, S. (1999). Channel estimation for ofdm systems with transmitter diversity in mobile wireless channels. IEEE J.Sel. Areas Commun, Vol.17, No.3, pp 461-471, ISSN: 0733-8716

Truman, T.E. \& Brodersen, R.W (1997). A measurement-based characterization of the time variation of an indoor wireless channel, proceedings of Int. Universal Personal Communications Record (ICUPC), pp. 25-32, ISBN: 0-7803-3777-8, San Diego, CA, USA, October 1997

Knopp, R. \& Humblet, P. (1995). Choi, J. (1996). Information capacity and power control in single-cell multiuser communications, proceedings of IEEE Conference on Communications (ICC), pp 331-335, ISBN: 0-7803-2486-2, Seattle, WA, USA, june 1995

Wong, C.Y.; Cheng, R.S.; Lataief, K.B. \& Murch, R.D. (1999). Multiuser OFDM with adaptative subcarrier, bit and power allocation. IEEE J.Sel. Areas Commun, Vol.17, No.10, pp 1747-1758, ISSN: 0733-8716

Wang, X. \& Xiang, Y. (2006). An OFDM-TDMA/SA MAC protocol with QoS constaints for broadband wireless LANs. ACM/Springer Wireless Networks, Vol. 12, No. 2, pp 159170, ISSN: 1022-0038

Viswanath, P.; Tse, D.N.C. \& Laroia, R. (2002). Opportunistic beamforming using dumb antennas. IEEE Transactions on Information Theory, Vol. 48, No.6, pp. 1277-1294, ISSN: 0018-9448

Kim, H.; Kim, K.; Han, Y. \& Lee, J. (2002). An efficient scheduling algorithm for QoS in wireless packet data transmission, proceedings of IEEE Int. Symposium on Personal, Indoor and Mobile Radio Communications (PIMRC), pp 2244-2248, ISBN: 0-7803-7589-0, Lisboa, Portugal, September 2002 
Anchun, W.; Liang, X.; Xjiin, S.X. \& Yan, Y. (2003). Dynamic resource management in the fourth generation wireless systems, proceedings of IEEE Int. Conference on Communication Technology (ICCT), pp 1095-1098, ISBN: 7-5635-0686-1, Beijing, China, April 2003

Svedman, P.; Wilson, K. \& Ottersen, B. (2004). A QoS-aware proportional fair scheduler for opportunistic OFDM, proceedings of IEEE Int. Vehicular Technology Conference (VTC), pp 558-562, ISBN: 0-7803-8521-7, Los angeles, CA, USA, September 2004

Kim, H.; Kim, K.; Han, Y. \& Yun, S. (2004). A proportional fair scheduling for multicarrier transmission systems, proceedings of IEEE Int. Vehicular Technology Conference (VTC), pp. 409-413, ISBN: 0-7803-8521-7, Los angeles, CA, USA, September 2004

Choi, J.-G. \& Bahk, S. (2007). Cell-throughput analysis of the proportional fair scheduler in the single-cell environment. IEEE Transactions on Vehicular Technology, vol. 56, No.2, pp. 766-778, ISSN: 0018-9545

Gueguen, C. \& Baey, S. (2008). Compensated proportional fair scheduling in multiuser OFDM wireless networks, proceedings of IEEE Wireless and Mobile Computing, Networking and Communications (WIMOB), pp119-125, ISBN: 978-0-7695-3393-3, Avignon, France, October 2008

Holtzman, J. (2001). Asymptotic analysis of proportional fair algorithm, proceedings of IEEE Int. Symposium on Personal, Indoor and Mobile Radio Communications (PIMRC), pp. 3337, ISBN: 0-7803-7244-1, San Diego, CA, USA, October 2001

Gueguen, C. \& Baey, S. (2008). Weighted fair opportunistic scheduling for multimedia QoS support in multiuser OFDM wireless networks, proceedings of IEEE Int. Symposium on Personal, Indoor and Mobile Radio Communications (PIMRC), pp. 1-6, ISBN: 978-14244-2643-0, Cannes, France, September 2008

Gueguen, C. \& Baey, S. (2008). An efficient and fair scheduling scheme for multiuser OFDM wireless networks, proceedings of IEEE Int.Wireless Communications and Networking Conference (WCNC), pp. 1610-1615, ISBN: 978-1-4244-1997-5, Las Vegas, NV, USA, April 2008

Gueguen, C. \& Baey, S. (2008). Scheduling in OFDM wireless networks without tradeoff between fairness and throughput, proceedings of IEEE Int. Vehicular Technology Conference (VTC), pp. 1-5, ISBN: 978-1-4244-1721-6, Calgary, Canada, September 2008

Gueguen, C. \& Baey, S. (2009). A Fair Opportunistic Access Scheme for Multiuser OFDM Wireless Networks. EURASIP Journal on Wireless Communications and Networking. Special issue on "Fairness in Radio Resource Management for Wireless Networks". Volume 2009 (2009), Article ID 726495, pp. 70-83

Parsons, J.D (1992). The Mobile Radio Propagation Channel, Wiley, ISBN: 978-0-471-98857-1

Baey, S. (2004). Modeling MPEG4 video traffic based on a customization of the DBMAP, proceedings of Int. Symposium on Performance Evaluation of Computer and Telecommunication Systems (SPECTS), pp. 705-714, ISBN: 1-56555-284-9, San Jose, California, USA, July 2004

Brady, P. (1969). A model for generating on-off speech patterns in two-conversation. Bell System Technical Journal, vol. 48, No.1, pp. 2445-2472 


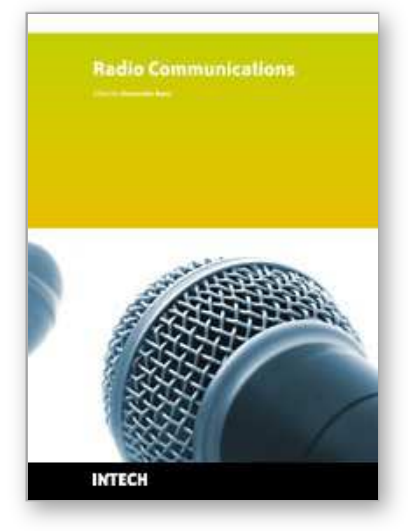

\author{
Radio Communications \\ Edited by Alessandro Bazzi
}

ISBN 978-953-307-091-9

Hard cover, 712 pages

Publisher InTech

Published online 01, April, 2010

Published in print edition April, 2010

In the last decades the restless evolution of information and communication technologies (ICT) brought to a deep transformation of our habits. The growth of the Internet and the advances in hardware and software implementations modified our way to communicate and to share information. In this book, an overview of the major issues faced today by researchers in the field of radio communications is given through 35 high quality chapters written by specialists working in universities and research centers all over the world. Various aspects will be deeply discussed: channel modeling, beamforming, multiple antennas, cooperative networks, opportunistic scheduling, advanced admission control, handover management, systems performance assessment, routing issues in mobility conditions, localization, web security. Advanced techniques for the radio resource management will be discussed both in single and multiple radio technologies; either in infrastructure, mesh or ad hoc networks.

\title{
How to reference
}

In order to correctly reference this scholarly work, feel free to copy and paste the following:

Cedric Gueguen and Sebastien Baey (2010). Opportunistic Access Schemes for Multiuser OFDM Wireless Networks, Radio Communications, Alessandro Bazzi (Ed.), ISBN: 978-953-307-091-9, InTech, Available from: http://www.intechopen.com/books/radio-communications/opportunistic-access-schemes-for-multiuser-ofdmwireless-networks

\section{INTECH}

open science | open minds

\author{
InTech Europe \\ University Campus STeP Ri \\ Slavka Krautzeka 83/A \\ 51000 Rijeka, Croatia \\ Phone: +385 (51) 770447 \\ Fax: +385 (51) 686166 \\ www.intechopen.com
}

\author{
InTech China \\ Unit 405, Office Block, Hotel Equatorial Shanghai \\ No.65, Yan An Road (West), Shanghai, 200040, China \\ 中国上海市延安西路65号上海国际贵都大饭店办公楼 405 单元 \\ Phone: +86-21-62489820 \\ Fax: +86-21-62489821
}


(C) 2010 The Author(s). Licensee IntechOpen. This chapter is distributed under the terms of the Creative Commons Attribution-NonCommercialShareAlike-3.0 License, which permits use, distribution and reproduction for non-commercial purposes, provided the original is properly cited and derivative works building on this content are distributed under the same license. 